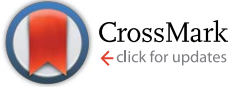

Cite this: RSC Adv., 2017, 7, 8927

Received 28th November 2016 Accepted 22nd January 2017

DOI: 10.1039/c6ra27465j

rsc.li/rsc-advances

\section{The effect of different hydrogen terminations on the structural and electronic properties in the triangular array graphene nanomeshes}

\author{
G. P. Tang, ${ }^{\star}$ Z. H. Zhang, ${ }^{\star}$ X. Q. Deng, Z. Q. Fan, H. L. Zhang and L. Sun
}

Constructing periodic nanoscale holes on graphene to form graphene nanomeshes (GNMs) is an effective way for opening band gaps. The effect of different hydrogen terminations on the structural and electronic properties in the triangular array graphene nanomeshes is investigated systematically from first-principles. The calculated results from the formation energy, Gibbs free energy, and phonon band structure reveal that the di-hydrogenated $\mathrm{sp}^{3}$ hybridization is the most favorable structure for the hole edges of GNMs except that the other hydrogen terminations may be stable under extremely-poor hydrogen conditions. The electronic properties of GNMs are very sensitive to edge hydrogen terminations, the GNM could be a semimetal, semiconductor, magnetic half-semimetal, or Bipolar Magnetic Semiconductor (BMS) by varying the edge hydrogen terminations, and the GNMs terminated by di-hydrogenation could open a sizable band gap due to the stronger on-site potential between holes, while the gap width could be tuned between semimetallic and semiconducting states by varying the neck width.

\section{Introduction}

As carbon nanomaterials of the two-dimensional honeycomb lattice, graphene has aroused tremendous research interests in the past decade. Graphene has a fairly simple atomic structure, a rather unique electronic structure, a high in-plane mobility of charge carrier, a long spin relaxation length and lifetime, and acts as an excellent ballistic spin transport channel. ${ }^{1-3}$ Therefore, it is predicted to be a promising material for nextgeneration high-performance nano-size electronic and spintronic devices, and the experimental work continues to make progress in applications. ${ }^{4-8}$ However, for pristine graphene, the semimetal nature (narrow band gap) limits its applications. Accordingly, enormous efforts have been devoted to open its band gap. ${ }^{9-12}$ For example, the approach of cutting twodimensional graphene into a one-dimensional graphene nanoribbon (GNR) has been proven efficiently in making a sizable band gap, and substitution of doping guest element into graphene could also open the band gap by breaking the sublattice equivalence, etc. However, these approaches are faced with some critical challenges because the manipulation is very difficult in experiments.

Recently, energetic investigations have been implemented on constructing periodic nanoscale holes on graphene, ${ }^{13-17}$ known as the graphene antidot lattice, that is the graphene nanomesh (GNM). GNM displays promising advantages relative

Institute of Nanomaterial and Nanostructure, Changsha University of Science \& Technology, Changsha 410114, China. E-mail: guipingtang@163.com; cscuzzh@ 163.com to existing graphene. Some techniques have been developed to fabricate and operate such nanomesh lattices, for example, electron-beam irradiation etching, ${ }^{18}$ oxygen reactive ion etching, ${ }^{19}$ atomic force microscopy etching, ${ }^{20}$ laser shock, ${ }^{21}$ block copolymer lithography, ${ }^{14,22}$ nanoimprint lithography, ${ }^{23}$ and nanosphere lithography. ${ }^{24}$ These experimental progresses in fabricating GNM shed light on the feasibility of GNM applied in nanodevices. More recently, the GNM-based field-effect transistor with superior on/off ratio was confirmed to support nearly 100 times greater currents than individual GNR device. ${ }^{14}$ Significant results have also been achieved in the theoretical investigations on GNMs. Theoretical calculations have predicted that lattice modification by perforating would realize a transition of properties of graphene from semimetallic to semiconducting, where the opened band gap could be tuned by the size, shape, density and symmetry of the perforated hole. ${ }^{13,17,25-34}$ For instance, Pedersen et al. found a simple scaling rule for band gap with respect to the numbers of removed and original total carbon atoms in a unit cell. ${ }^{13}$ But previous theoretical works have not sufficiently considered the arrays of perforated holes in graphene, especially, another interesting issue about different terminations of the hole edges affecting the structural stability and the electronic properties of GNM has been less addressed. From the view of both fundamental and application studies, it is worthy of detailed studies for these cases.

In fact, there are several array methods of holes which can ensure the all holes periodically aligned in the GNM system, the two most conventional arrays among them are the rectangular array and the triangular array as shown in Fig. 1, and the 
(a)
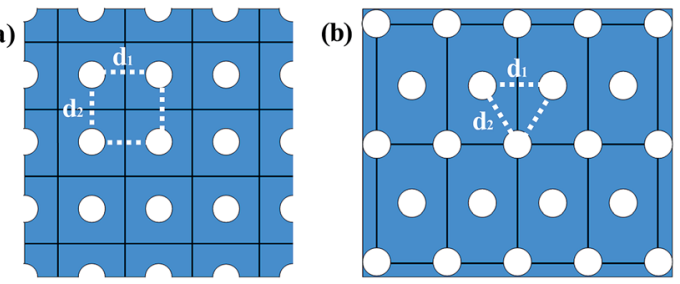

Fig. 1 The schematic diagram of GNM with (a) rectangular array and (b) triangular array of holes. Blue area represents graphene sheet, white circles denote the holes, and black straight lines are their boundaries representing the unit cell, $d_{1}$ and $d_{2}$ represent the distances between neighbor holes.

triangular array of holes is the most likely generate form of experimental techniques such as block copolymer lithography, nanoimprint lithography, and nanosphere lithography. The electronic structures of the rectangular array GNMs have investigated in the previous work. ${ }^{13,15,34}$ In the present work, based on the first principles calculations, we systematically investigate the structural and electronic properties of the triangular array GNM, in particular, the effect on properties due to the various hydrogen terminations of the hole edges. Meanwhile, we analyze in depth the fundamental mechanism for the intriguing properties. We believe that our findings can benefit further theoretical and experimental studies on the graphenebased nanostructures.

\section{Models and methods}

As illustrated in Fig. 1, the GNM system can be characterized by $d_{1}, d_{2}$ and $r$, where $d_{1}$ and $d_{2}$ represent the distances between neighbor nanoholes (i.e. neck widths) along the length and the other orientations, respectively, $r$ represents the radius of nanohole. The GNM systems contain triangular arrays of hole, which all consist of the repeating rectangular unit cell shown as the area surrounded by one black rectangle in Fig. 1(b). One unit cell contains two holes (one hole plus four quarter of one hole). We only consider quasi-circle shapes of the perforated hole since it is the most conventional and realistic shape hole experimentally. Consequently, the unit cells of these GNMs can be described by the parameters $M, N$ and $R$ as illustrated in Fig. 2(a). Here $M$ and $N$ indicate the periodicities of unit cell for the graphene matrix along the zigzag and armchair edge orientations, respectively. All the above parameters can be expressed with the graphene lattice constant $a_{0}=\sqrt{3} a_{\mathrm{cc}}=2.46 \AA$, where $a_{\mathrm{cc}}=1.42 \AA$ is the bond length between neighboring carbon atoms. Furthermore, there exists a well-defined relationship between the geometrical parameters of GNM and its unit cell parameters as follow: $r=\frac{1}{2}(R+1) a_{0}$, $d_{1}=(M-2 r) a_{0}=(M-R-1) a_{0}$, and $d_{2}=\frac{1}{2}\left[\left(M^{2}+3 N^{2}\right)^{1 / 2} a_{0}-4 r\right]=\left[\frac{1}{2}\left(M^{2}+3 N^{2}\right)^{1 / 2}-R-1\right] a_{0}$.

The periodically perforating holes on graphene matrix, can arise a dangling bond for each carbon atom of the hole edges. It is confirmed that the GNM with unpassivated holes edges can exist in super vacuum, ${ }^{35}$ but the dangling bonds of carbon atoms of the hole edges are easy to be saturated with various ways once the GNM is exposed to environment. For example, hydrogenation is the simplest way to realize. At an extremelylow hydrogen-concentration, the hole edges are generally terminated with one hydrogen atom per carbon atom to form a $\mathrm{sp}^{2}$-type topology, namely, mono-hydrogenation. In contrast, under a rich hydrogen-concentration, the hole edges are probably terminated with two hydrogen atoms per carbon atom, known as di-hydrogenation inducing $\mathrm{sp}^{3}$ hybridization. And there is a possible to form a mono- and di-hydrogenated composite type for a properly hydrogen-concentration. In order to address conveniently, in term of the hydrogenconcentration from a low value to a high value, we select six hydrogen terminations for the hole edge of the GNM include unpassivation, mono-hydrogenation, three mono- and dihydrogenated composite types, and di-hydrogenation, which are denoted as $0-\mathrm{H}, 1-\mathrm{H}, 211-\mathrm{H}, 21-\mathrm{H}, 221-\mathrm{H}$, and $2-\mathrm{H}$, respectively. The corresponding structural schemes are shown in Fig. 2(b).

We consider well-fledged first-principle calculations using the density functional theory (DFT) which can provide the most detailed and accurate description of the electronic structure. According to the theory of KS (Kohn and Sham), the ground
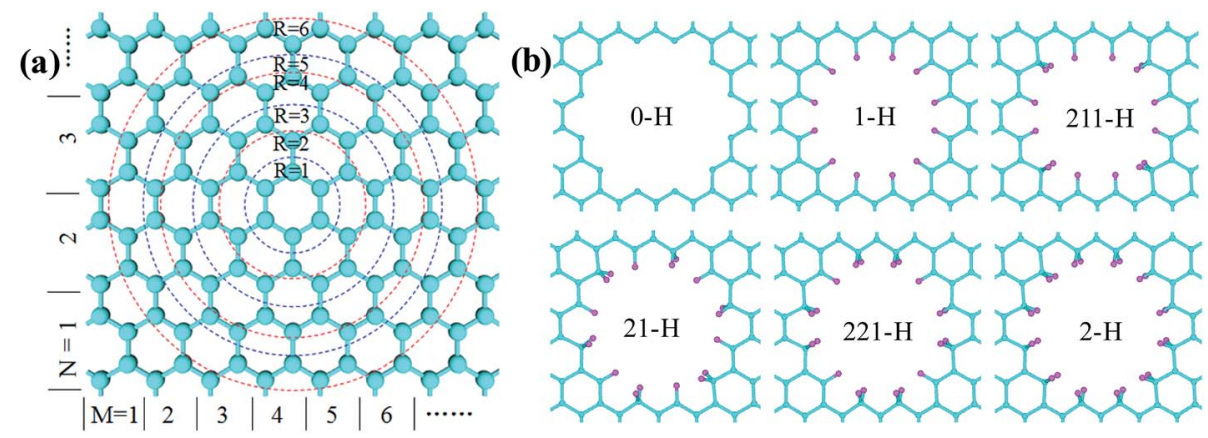

Fig. 2 (a) The geometries and parameter $R$ of circular hole in GNM lattices. $R$ is an integer and becomes bigger in turn from inside to outside when more carbon atoms are removed. Here $M$ and $N$ are the parameters of unit cells; indicate the periodicities along the zigzag and armchair lattice orientations, respectively. (b) The geometry structures of the hydrogen-terminated edges in GNM. The cyan (pink) spheres represent $\mathrm{C}(\mathrm{H})$ atoms. 
state energy is uniquely determined by the ground state electron density, density can be obtained from a single-particle picture of non-interacting electrons. The corresponding Hamiltonian for the single-particle KS orbitals $\psi_{i}$ is expressed by the KS equations as

$$
H^{\mathrm{KS}} \psi_{i}(r)=\left[-\frac{1}{2} \nabla^{2}+V_{\mathrm{eff}}(r)\right] \psi_{i}(r)=\varepsilon_{i} \psi_{i}(r)
$$

where the effective potential

$$
V_{\text {eff }}(r)=\int d r^{\prime} \frac{\rho\left(r^{\prime}\right)}{\left|r-r^{\prime}\right|}+V_{\mathrm{a}}\left(r,\left\{R_{i_{\mathrm{a}}}\right\}\right)+\frac{\delta E_{\mathrm{XC}}[\rho(r)]}{\delta \rho(r)}
$$

depends explicitly on the density $\rho(r)=\sum_{i_{0}}\left|\psi_{i}(r)\right|^{2}$ with the sum running over occupied KS orbitals. Here, $V_{\mathrm{a}}\left(r,\left\{R_{i_{3}}\right\}\right)$ is the external potential due to the atoms at positions $R_{i_{a}}$. The socalled exchange-correlation term $E_{\mathrm{XC}}(r)$ accounts for all manybody effects and is not known exactly, but must be appropriately approximated. Finally, the ground state energy of the interacting problem is

$$
\begin{aligned}
E[\rho(r)]= & T[\rho(r)]+\int \mathrm{d} r \rho(r) V_{\mathrm{a}}\left(r,\left\{R_{i_{\mathrm{a}}}\right\}\right)+\frac{1}{2} \iint \mathrm{d} r \mathrm{~d} r^{\prime} \frac{\rho(r) \rho\left(r^{\prime}\right)}{\left|r-r^{\prime}\right|} \\
& +E_{\mathrm{XC}}[\rho(r)]
\end{aligned}
$$

where $T$ is the kinetic energy corresponding to the density $\rho(r)$, which is a function of only three coordinates.

In this work, the geometry optimization and electronic structure are calculated by using DFT as implemented in Atomistix ToolKit (ATK). The generalized gradient approximation (GGA) and the single-zeta polarized basis set (SZP) are full used; the local density approximation (LDA) and the meta generalized gradient approximation (MGGA) are occasionally used for comparing. To examine the accuracy and completeness of the results, we have also performed calculations using spinpolarized DFT (SGGA) for the typical cases. The convergence criteria of force and energy are set to be $0.01 \mathrm{eV} \AA^{-1}$ and $1 \times$ $10^{-3} \mathrm{eV}$, respectively, and the electron temperature is fixed at $300 \mathrm{~K}$.

\section{Results and discussion}

\subsection{Structural stability of GNM}

We first investigate the structural stability of the GMM with different edge hydrogen terminations; the stability is normally determined by the formation energy. GNM segments in one rectangular unit cell consist of $n_{\mathrm{C}}$ carbon atoms and $n_{\mathrm{H}}$ hydrogen atoms. The segment formation energy per area is then calculated using:

$$
E_{\mathrm{S}}=\left(E_{\text {total }}-n_{\mathrm{C}} E_{\mathrm{C}}-n_{\mathrm{H}} E_{\mathrm{H}}\right) / A
$$

here, $E_{\text {total }}$ is the total internal energy of the GNM segment in a unit cell, $A$ is the area of one unit cell, $E_{\mathrm{C}}$ and $E_{\mathrm{H}}$ are the energies of an isolated carbon and hydrogen atom, respectively. And the segment hydrogen and carbon density per area is also calculated by $\rho_{\mathrm{H}}=n_{\mathrm{H}} / A$ and $\rho_{\mathrm{C}}=n_{\mathrm{C}} / A$, respectively. We investigate two typical GNM segments as one for $R=1, M=6, N=4$; another for $R=3, M=9, N=5$. The calculated formation energies, hydrogen densities and carbon densities per area are illustrated as Table 1.

We can find the formation energies per area $\left(E_{\mathrm{S}}\right)$ of both GNM segments become lower as increasing the numbers of $\mathrm{H}$ atoms terminated around the hole edges, and the maximum and minimum of $E_{\mathrm{S}}$ occur in the case of an unpassivation $(0-\mathrm{H})$ and di-hydrogenation $(2-\mathrm{H})$, respectively. It indicates the unpassivated GNMs are not stable enough, the structural stabilities of GNMs are gradually enhanced with the increase of the number of terminated $\mathrm{H}$ atoms, and the structures are the most stable when the hole edges of GNMs are di-hydrogenated. It can be also found the $E_{\mathrm{S}}$ of the former (GNM for $R=1, M=6$, $N=4$ ) is lower than that of the latter (GNM for $R=1, M=6, N=$ 4 ) with the same hydrogen termination. It suggests the former structure is more stable than the later, which can be understood from the later one having a smaller $\rho_{\mathrm{C}}$ due to the bigger perforated hole.

The above calculations are only considered at a zerotemperature ideal condition, but the formation energy per area $E_{\mathrm{S}}$ can be used to determine the stability of different structures as a function of the experimental conditions, one reason is that the chemical potential of hydrogen is variable in experiment. In order to consider a molecular hydrogen gas atmosphere around the GNM edge, the relative stability is generally obtained by comparing the Gibbs free energies of systems: $:^{36,37}$

$$
G_{\mathrm{H}_{2}}=E_{\mathrm{S}}-\frac{1}{2} \mu_{\mathrm{H}_{2}} \rho_{\mathrm{H}}
$$

here, $\mu_{\mathrm{H}_{2}}$ is the chemical potential of hydrogen, which depends on the pressure and temperature of the system and directly reflects the hydrogen-concentration in the system. We calculate and compare the Gibbs free energies for six different structures under $R=3, M=9, N=5: 0-\mathrm{H}, 1-\mathrm{H}, 211-\mathrm{H}, 21-\mathrm{H}, 221-\mathrm{H}$, and 2H. $G_{\mathrm{H}_{2}}$ as a function of the chemical potential $\mu_{\mathrm{H}_{2}}$ is plotted in Fig. 3(a). As can be seen, the structural stability of the GMM with different hydrogen-concentrations is distinctly different. In the

\begin{tabular}{|c|c|c|c|c|c|c|}
\hline & \multicolumn{3}{|c|}{$R=1, M=6, N=4$} & \multicolumn{3}{|c|}{$R=3, M=9, N=5$} \\
\hline & $\begin{array}{l}\rho_{\mathrm{C}} \\
\left(\AA^{-2}\right)\end{array}$ & $\begin{array}{l}\rho_{\mathrm{H}} \\
\left(\AA^{-2}\right)\end{array}$ & $\begin{array}{l}E_{\mathrm{S}} \\
\left(\mathrm{eV} \AA^{-2}\right)\end{array}$ & $\begin{array}{l}\rho_{\mathrm{C}} \\
\left(\AA^{-2}\right)\end{array}$ & $\begin{array}{l}\rho_{\mathrm{H}} \\
\left(\AA^{-2}\right)\end{array}$ & $\begin{array}{l}E_{\mathrm{S}} \\
\left(\mathrm{eV} \AA^{-2}\right)\end{array}$ \\
\hline $0-\mathrm{H}$ & 0.3333 & 0.0000 & -3.0911 & 0.2797 & 0.0000 & -2.5459 \\
\hline $1-\mathrm{H}$ & 0.3333 & 0.0476 & -3.3940 & 0.2797 & 0.0508 & -2.8767 \\
\hline $211-\mathrm{H}$ & 0.3333 & 0.0635 & -3.4525 & 0.2797 & 0.0678 & -2.9420 \\
\hline $21-\mathrm{H}$ & 0.3333 & 0.0714 & -3.4760 & 0.2797 & 0.0763 & -2.9741 \\
\hline 221-H & 0.3333 & 0.0794 & -3.5150 & 0.2797 & 0.0848 & -3.0018 \\
\hline $2-\mathrm{H}$ & 0.3333 & 0.0952 & -3.5792 & 0.2797 & 0.1017 & -3.0587 \\
\hline
\end{tabular}
region $\mathrm{Z} 1\left(\mu_{\mathrm{H}_{2}} \leq-7.49 \mathrm{eV}\right)$, namely, under extremely-poor hydrogen conditions, $1-\mathrm{H}$ is a stable structure. However, when a slightly sufficient hydrogen environment leads to the $\mu_{\mathrm{H}_{2}}$

Table 1 Formation energies, hydrogen densities and carbon densities per area for two typical GNM segments 

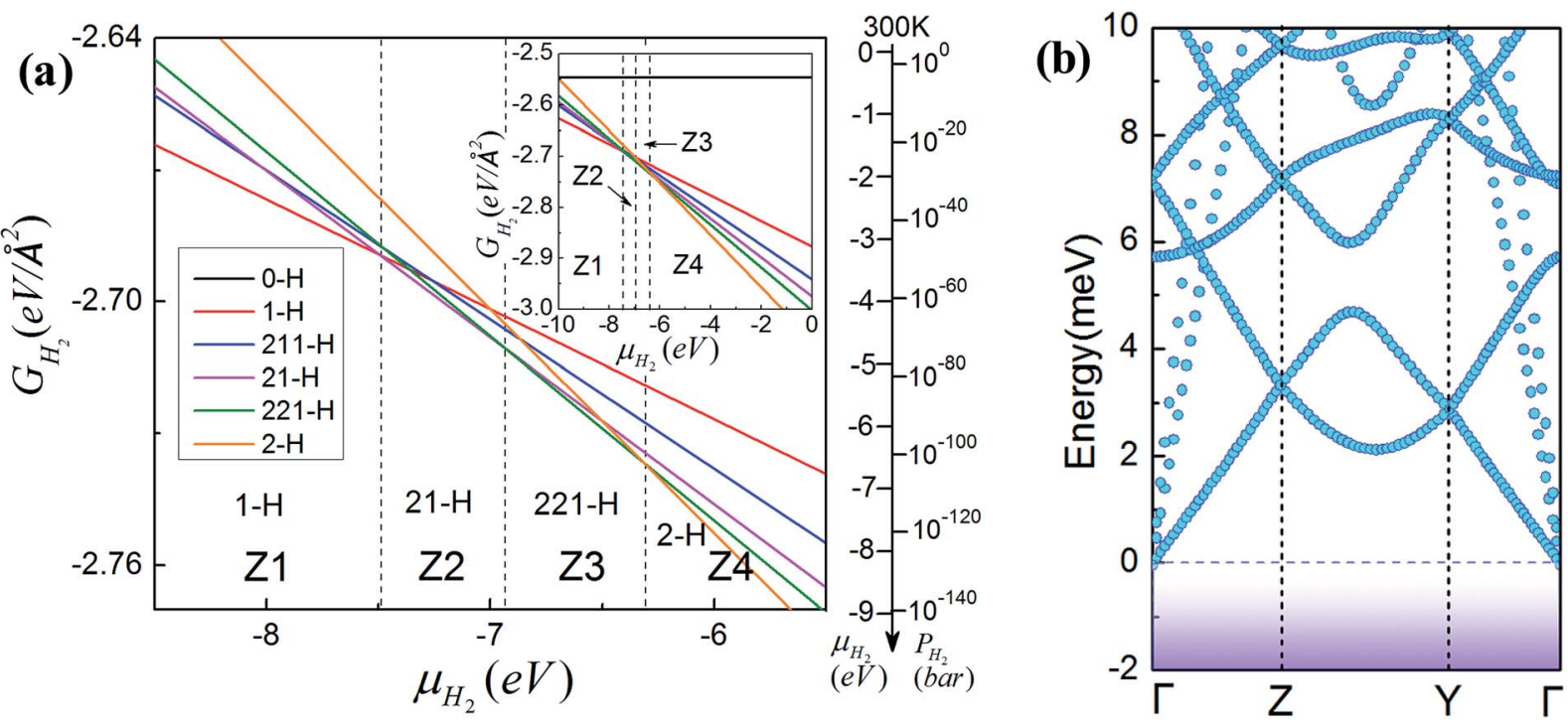

Fig. 3 (a) Gibbs free energy $G_{\mathrm{H}_{2}}$ versus chemical potential $\mu_{\mathrm{H}_{2}}$ for GNM with different edge hydrogen terminations. The right upper inset shows a larger range. The alternative right axis shows the pressure of molecular $\mathrm{H}_{2}$ gas corresponding to the chemical potentials at $T=300 \mathrm{~K}$ (data adapted from ref. 36). (b) Phonon band structure of GNM terminated with di-hydrogenation for $R=1, M=6, N=4$. It can be observed clearly that there are no negative bands.

enhancement, that is, in the quite large region $\mathrm{Z} 4\left(\mu_{\mathrm{H}_{2}} \geq-6.28\right.$ $\mathrm{eV}$ ), 2- $\mathrm{H}$ becomes energetically the most favorable structure. Only at reasonable hydrogen-concentration, $21-\mathrm{H}$ and $221-\mathrm{H}$ can stably exist in tiny regions $\mathrm{Z} 2\left(-7.49 \mathrm{eV}<\mu_{\mathrm{H}_{2}}<-6.86 \mathrm{eV}\right)$ and Z3 $\left(-6.86<\mu_{\mathrm{H}_{2}}<-6.28 \mathrm{eV}\right)$, respectively.

To examine the structural stability of GNM terminated with di-hydrogenation, we have calculated the phonon band structure of the typical GNM with 2-H termination for $R=1, M=6, N$ $=4$. The calculated phonon band structure is shown in Fig. 3(b). It can be observed clearly that there are no negative bands under the zero energy level, namely, no phonon imaginary frequencies appear. This just confirms that GNM with $2-\mathrm{H}$ termination is a stable configuration. It is worth noting that there are obvious differences in the structural stability between the twodimensional GNM and one-dimensional GNR terminated by hydrogen. ${ }^{36,37}$

\subsection{Electronic properties of GNM}

We now turn to explore the electronic properties of GNM with different edge hydrogen terminations. For the consideration of computing cost, we only select the GNM for $R=1, M=6$, and $N$ $=4$ as the prototype for detailed studies. A number of GNMs with other parameters such as $R=3, M=9$, and $N=5$ have also been adopted to examine our conclusions. The calculated energy band structures are shown in Fig. 4. It is clear that the band structures of GNM are very sensitive to edge hydrogen terminations, as more complex edge terminations should result in dramatic changes of the electronic structure. ${ }^{38}$

3.2.1 Electronic structure with magnetism. For $0-\mathrm{H}$ termination, that is an unpassivation, the energy bands are all split obviously regardless of $\alpha$ or $\beta$ spin subbands, and the band gap for $\alpha$ spin is about $0.95 \mathrm{eV}$ but that for $\beta$ spin is only $0.06 \mathrm{eV}$ ( $c f$. Fig. 4(a)). This indicates the unpassivated GNM is a magnetic half-semimetal. The unpassivated edge carbon atoms of one hole has six dangling bonds, each dangling bond introduces one Bohr magneton, $1 \mu_{\mathrm{B}}$, giving a total magnetization of $12 \mu_{\mathrm{B}}$ per unit cell, just the net magnetic moment causes the spin degeneracy to be broken down and the flat bands near the Fermi level arising from dangling bonds to depart from the Fermi level. Similarly to $0-\mathrm{H}$ termination, the energy bands are all split for 21-H termination too, but there is a substantial band gap for both $\alpha$ spin and $\beta$ spin ( $c f$. Fig. 4(d)). It is called a Bipolar Magnetic Semiconductor (BMS) or Bipolar Spin Semiconductor (BSS). BMS may be of great use in the next generation of information storage owing to the extremely low power consumption and the fast response time. Nevertheless, the split energy bands for $21-\mathrm{H}$ is not due to dangling bonds as the case of $0-\mathrm{H}$, since di-hydrogenated $\mathrm{sp}^{3}$ hybridization induce unequal numbers of A- and B-sublattice carbon atoms being saturated, according to Lieb's theorem, ${ }^{39}$ magnetic moment $M=\left|N_{\mathrm{A}}-N_{\mathrm{B}}\right|$ $\mu_{\mathrm{B}}$, here $N_{\mathrm{A}}$ and $N_{\mathrm{B}}$ denote the number of carbon atoms in $\mathrm{A}$ and B sublattices, respectively; so which should bring a net magnetic moment of $6 \mu_{\mathrm{B}}$ per unit cell, this magnetization is entirely due to the sublattice imbalance and does not come from dangling bonds. Of course, it is worth noting that the $21-\mathrm{H}$ termination doesn't always break the balance of sublattice with varying the size of hole. For clarity and to aid visualization, we have calculated and plotted the distribution of magnetism of GNM for both $0-\mathrm{H}$ and $21-\mathrm{H}$ in Fig. 5 . We can find that the magnetic moments for 21- $\mathrm{H}$ are evenly distributed on 24 carbon atoms and reside mainly on the $\pi$-orbitals, differently from the magnetic ordering for $0-\mathrm{H}$, which are strongly localized at the sites of the dangling bonds and involve only the $\mathrm{sp}^{2}$-orbitals; and the calculated results of the net magnetic moment for both $0-\mathrm{H}$ and $21-\mathrm{H}$ are in good agreement with the theoretical analysis. 


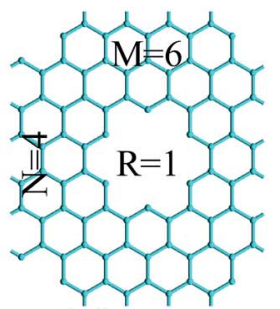

(a) 0 - $\mathrm{H}$
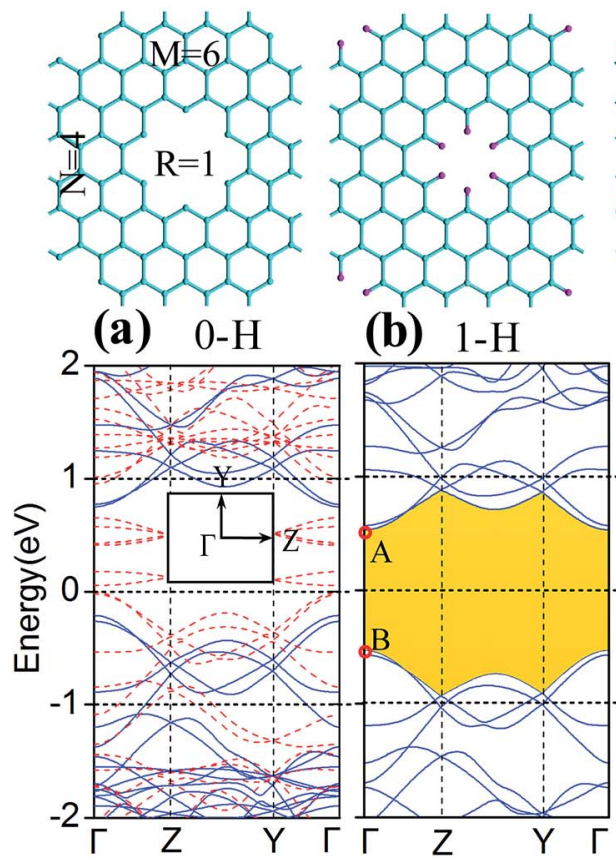

(b) $1-\mathrm{H}$ the Fermi level.

Fig. 4 Geometry structures and energy band structures of GNM with different edge hydrogen terminations for $R=1, M=6, N=4$. (a) $-(\mathrm{f}$ ) Represent the cases for $0-\mathrm{H}, 1-\mathrm{H}, 211-\mathrm{H}, 21-\mathrm{H}, 221-\mathrm{H}$, and $2-\mathrm{H}$, respectively. The upper models indicate the geometry structures of the corresponding unit cells, the cyan (pink) spheres represent $\mathrm{C}(\mathrm{H})$ atoms. In the lower band structures, $Y$ and $Z$ in (a) are symmetric $k$-point denoting wave vectors parallel to armchair and zigzag axis of GNM, respectively; band gaps in (b) and (f) are shaded yellow; $A$ in (b), $C$ in (e), and $E$ in ( $f$ ) are the points of conduction band minimum (CBM); $B$ in (b), $D$ in (e), and $F$ in ( $f$ ) are the points of valence band maximum (VBM); zero energy is set at
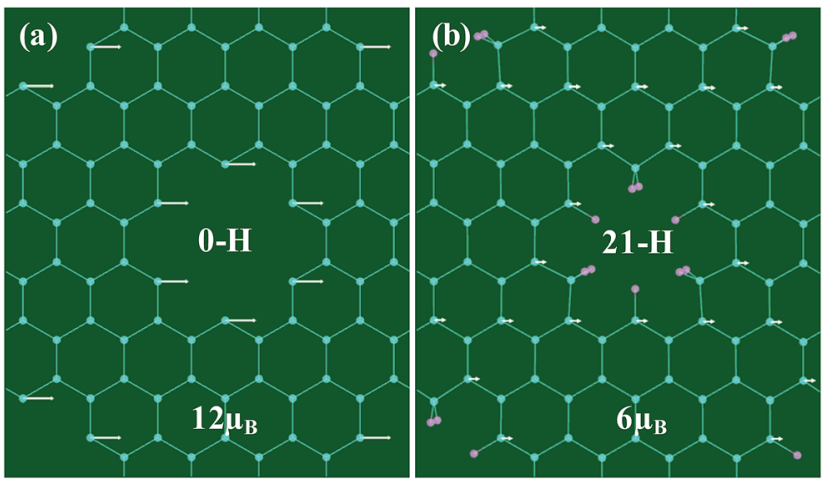

Fig. 5 The distribution of magnetism of GNM for (a) $0-\mathrm{H}$ and (b) $21-\mathrm{H}$. The white arrows represent the net magnetic moments located at the sites of carbon atoms. The total net magnetic moments of GNM for 0$\mathrm{H}$ and $21-\mathrm{H}$ are $12 \mu_{\mathrm{B}}$ and $6 \mu_{\mathrm{B}}$ per unit cell, respectively.

3.2.2 Electronic structure without magnetism. As can be seen from Fig. 4(b), (c), (e) and (f), for 1-H, 211- $\mathrm{H}$, and $2-\mathrm{H}$, the energy bands all are completely spin-degenerate. This means they all are non-magnetic. We can further find that both the GNM with 211-H termination and the case for $221-\mathrm{H}$ are semimetals with a narrowed band gap, the case for both $1-\mathrm{H}$ and $2-\mathrm{H}$ is semiconductors, and the band gap of GNM with 2-H termination is slightly larger than the case for $1-\mathrm{H}$.

To understand the impacts of $1-\mathrm{H}, 211-\mathrm{H}$, and $2-\mathrm{H}$ terminations on electronic structures, we have calculated the Bloch states at six points of CBM and VBM (i.e. A, B, C, D, E, and F shown in Fig. 4) and the corresponding partial density of states (PDOS) as shown in Fig. 6 and 7, respectively.

In Fig. 6(b), the Bloch states at both $\mathrm{C}$ and $\mathrm{D}$ are delocalized enough to form electron transmission channels along the $Z$ axis orientation while there is no channel due to the localization of Bloch states along the $Y$ axis orientation. As a result, the GNM for 221-H possesses a very narrow band gap along the $I-Z$ direction while a wide band gap along the $\Gamma-Y$ direction ( $c f$. Fig. 4(e)). In Fig. 6(a) and (c), the Bloch states at A, B, E, and F are all localized so as to no electron transmission channel either $Z$ or $Y$ axis orientation, and the localization for $2-\mathrm{H}$ is slightly stronger than that for 1- $\mathrm{H}$, especially, near the hole edge, Bloch wave for 2-H almost disappears compared with $1-\mathrm{H}$. Consequently, the band gap of GNM with $2-\mathrm{H}$ termination is slightly larger than the case for 1-H (cf. Fig. 4(b) and (f)).

In Fig. 7(a)-(c), the energy gaps, namely, the energy ranges of DOS maintaining at zero around the Fermi level, are well consistent with the band gaps in Fig. 4(b), (e) and (f), respectively. And just the DOS peak does not appears at Fermi level, but rather a narrow energy gap, so the GNM for $221-\mathrm{H}$ is semi metallic rather than metallic. We can also find comparing the Fig. 7(a) and (c), the PDOS of $\mathrm{H}$ atoms for $2-\mathrm{H}$ is a little stronger than that for $1-\mathrm{H}$ as the number of $\mathrm{H}$ atoms for $2-\mathrm{H}$ is double number for $1-\mathrm{H}$; but the PDOS of $\mathrm{C}$ atoms terminated by hydrogen atoms for 2- $\mathrm{H}$ is significantly weaker than that for 1$\mathrm{H}$. These lead to a larger energy gap for GNM with dihydrogenation. 

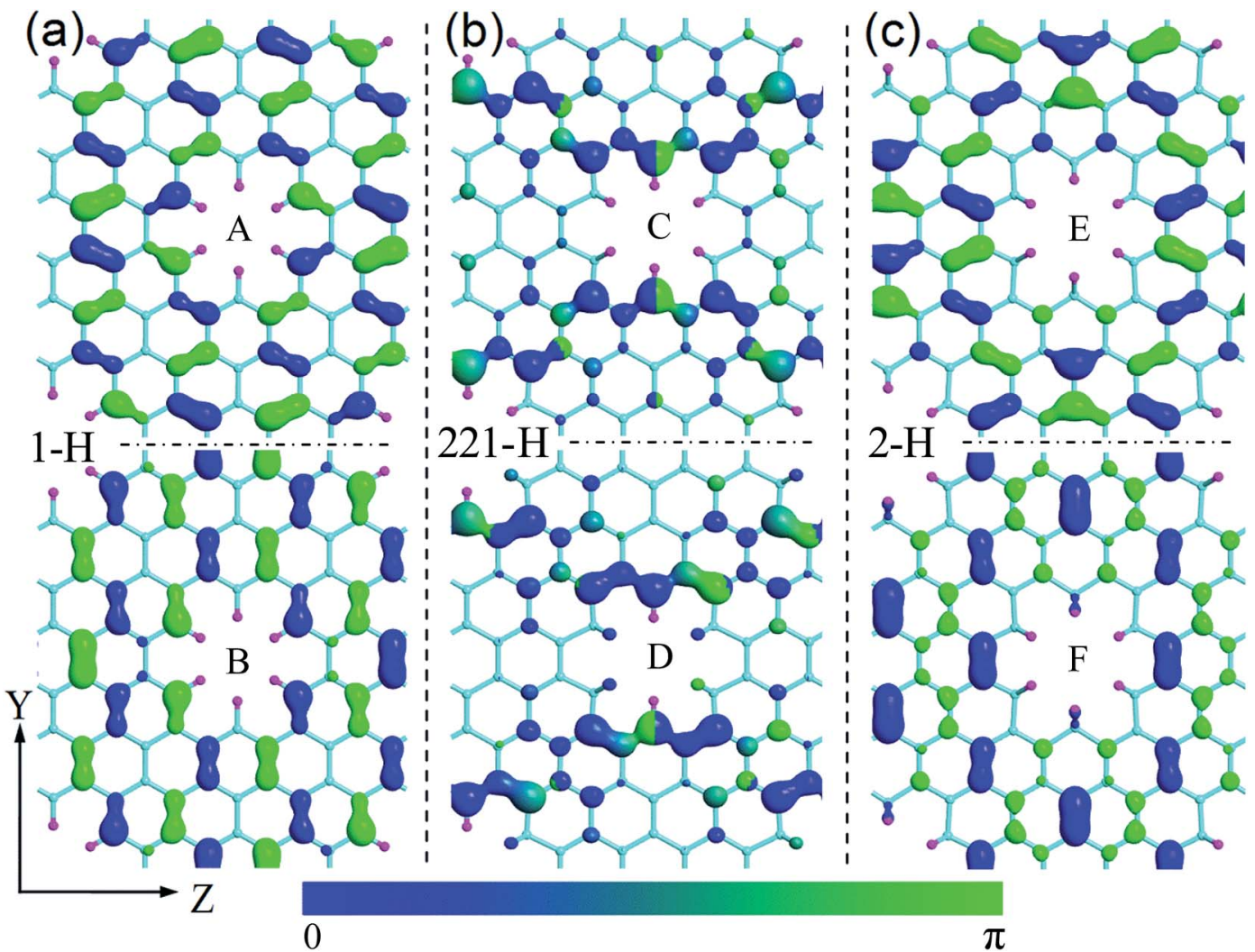

Fig. 6 Bloch states at six points of CBM and VBM. (a)-(c) Correspond to the cases for 1-H, 221-H, and 2-H, respectively. A, B, C, D, E, and F just are the six points shown in Fig. 4. The isosurface value is fixed at 0.025 a.u. $Y$ and $Z$ are two coordinate axes along the armchair and zigzag lattice orientations of GNM, respectively.

In the following, we further explore the underlying mechanisms for opening energy gap by mono- and di-hydrogenation. The introduction of periodic holes in graphene will certainly bring a perturbative on-site potential,$^{38}$ causing wave functions to degenerate at the hole edge and to change the energy dispersion. Therefore, one common origin of energy gap for GNMs is the quantum-confinement effect due to the on-site potential. Furthermore, the on-site potential is very sensitive to boundary conditions such as mono- and di-hydrogenated terminations. We have calculated the on-site potential of one
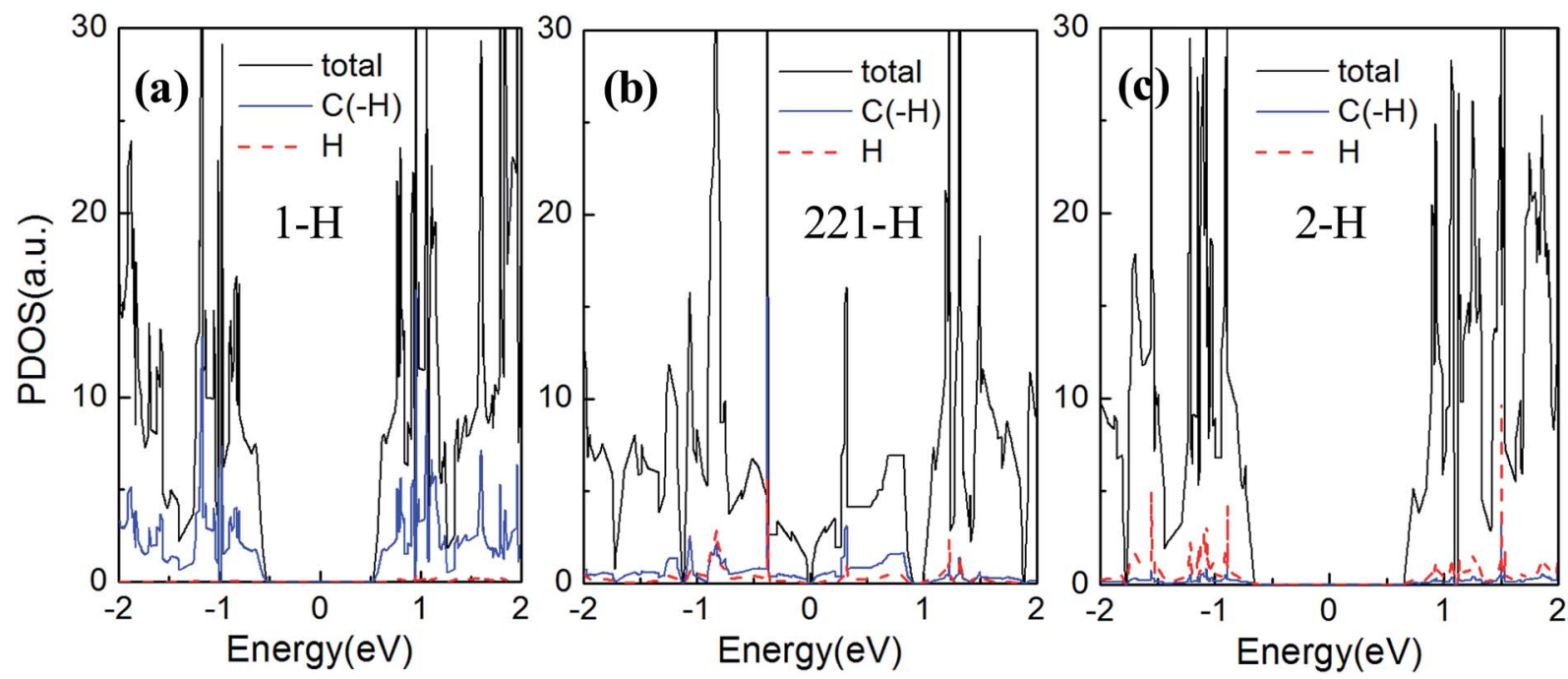

Fig. 7 The total density of states (black solid line) and partial densities of states of hydrogen atoms (red dashed line) and carbon atoms terminated by hydrogen atoms (blue solid line) in one unit cell. The (a)-(c) are for 1- $\mathrm{H}, 221-\mathrm{H}$, and 2- $\mathrm{H}$ terminations, respectively. 
unit cell of GNM for $R=1, M=6, N=4$, and have projected it on $Y$ and $Z$ axes for comparing. The calculated results are shown in Fig. 8. It can be clearly found the on-site potential between neighbor holes is much stronger than that of hole whether for 2$\mathrm{H}$ or for $1-\mathrm{H}$, and the on-site potential between holes for $2-\mathrm{H}$ is some stronger than the case for 1- $\mathrm{H}$ whether on $Y$ or on $Z$ axis, just the on-site potential exhibits gravitational interaction to electrons, which causes the electron wave functions of hole edge states to be degenerate for $1-\mathrm{H}$ and $2-\mathrm{H}$, and the case for $2-\mathrm{H}$ to be more degenerate than the case for 1-H (cf. Fig. 6(a) and (c)). As a consequence, the energy gap for 2-H is larger than that for 1-H (cf. Fig. 4(b), (f) and 7(a), (c)).

\subsection{Energy-gap altering regularities of GNM}

We finally investigate the altering regularities of the energy (band) gap of GNM. We only consider the GNM terminated with mono- and di-hydrogenation owing to their structures being stable. As stated above, the unit cell of GNM is described by three parameters: $R, M$, and $N$. For the convenience of study, we change one parameter and fix the other two parameters for the value of $M, N$, and $R$ in sequence. Increasing the value of $M$ is equivalent to increase the distance between neighboring holes along the zigzag lattice direction, and similarly, increasing the value of $N$ is to increase the distance between neighboring holes along the armchair lattice direction. The GNMs for $R=1,3$, and 4 are chosen as examples and the calculated results are plotted in Fig. 9.

As can be seen from Fig. 9(a) and (b), for the GNMs terminated with mono- or di-hydrogenation, the band gap displays an oscillatory decrease with the increasing parameter $M$ by a periodicity of 6 at the fixed $R$ and $N$, this is different from the oscillation periodicity of 3 for the rectangular array $\mathrm{GNM} ;{ }^{34}$ the necessary condition is not a sufficient condition to generate a maximal band gap is $M=3 p$ ( $p$ is a positive integer). It suggests that varying $M$ would lead to a variation between semimetallic and semiconducting behaviors. This case demonstrates that the periodicity along the zigzag edge orientation of GNM also plays crucial role in band gap opening, in addition to the quantum-
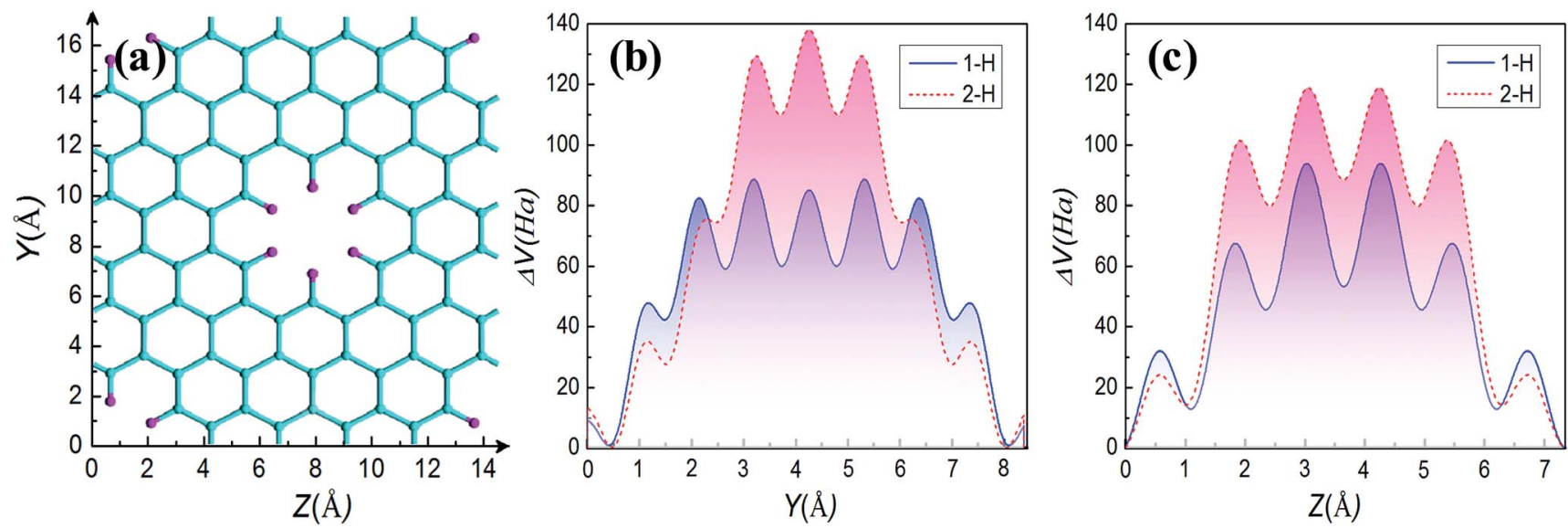

Fig. 8 The projected distribution of on-site potential $\Delta V$ of GNM for mono-hydrogenation (blue solid line) and di-hydrogenation (red dashed line). (a) Shows $Y$ and $Z$ are two projection axes along the armchair and zigzag lattice orientations of GNM, respectively. (b (c)) Shows one periodic unit of projected distribution of on-site potential on $Y(Z)$ axis. Zero potential energy is set at the center of holes.
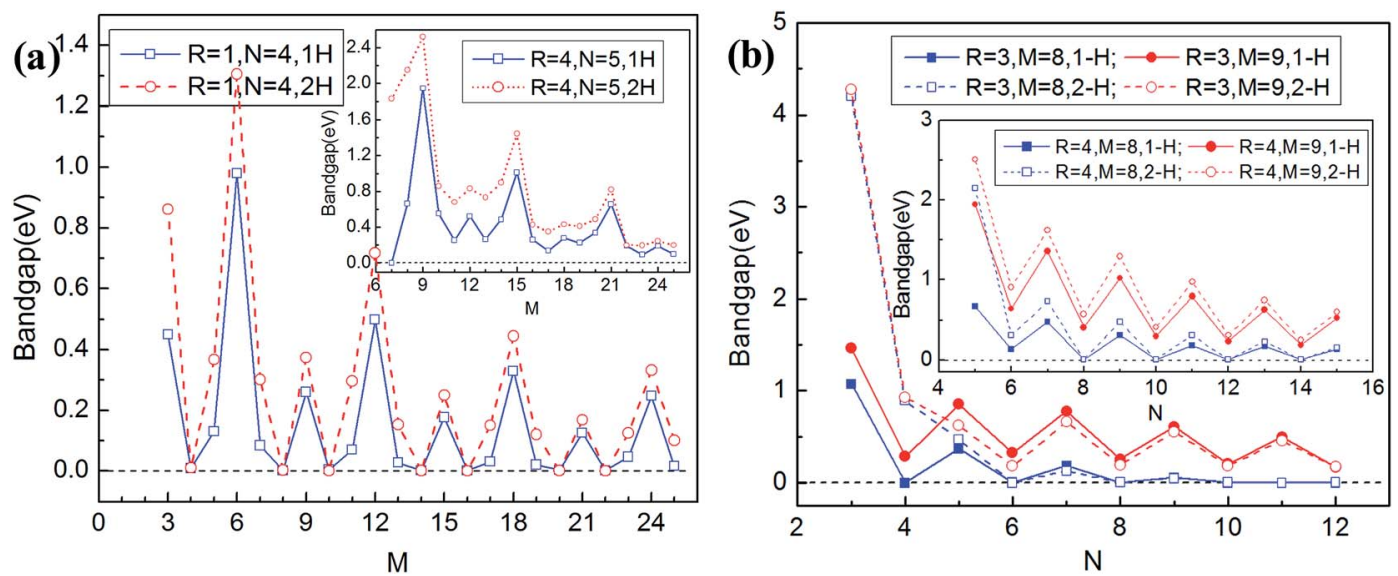

Fig. 9 The band gap of GNM terminated with mono- (solid line) and di-hydrogenation (dashed line) as a function of structural parameter $M, N$ and $R$. 
confinement. And it can be found that if the parameter $M$ and $R$ remain unchanged while only change parameter $N$, the band gap displays an odd-even oscillatory effect, the band gaps for $N=$ odd are the tops of the oscillation plotlines while the cases for $N$ $=$ even are the bottoms. It can be also noted that the band gap decrease to a steady nonzero value gradually with the increasing parameter $N$ while $M=3 p$, but it drop to zero rapidly with the increasing parameter $N$ while $M \neq 3 p$. Furthermore, for a specific perforated hole, the bigger the unit cell size is, the smaller the band gap would be, as the bigger unit cell size corresponds to the wider neck width and the lower hole density correspondingly. In short, the band gap could be opened in the GNM with size $M=3 p$, and it could be tuned by varying the neck width or edge terminations. This is greatly useful for the experimental investigations. In addition, it is worth noting that the band gap of the triangular array GNM is not proportional to the size of hole similar to the rectangular array GNM.

\section{Conclusions}

Based on the first-principles method, we systematically investigate the effect of different hydrogen terminations on the structural and electronic properties in the triangular array graphene nanomeshes. We firstly calculated the formation energy $E_{\mathrm{S}}$ under zero-temperature ideal condition and the Gibbs free energy $G_{\mathrm{H}_{2}}$ under room-temperature general condition, respectively. The both calculated results reveal that the dihydrogenated $(2-\mathrm{H}) \mathrm{sp}^{3}$ hybridization is the most favorable structure for the hole edges of GNM except that the other hydrogen terminations may be stable under extremely-poor hydrogen conditions. And the GNM with $2-\mathrm{H}$ termination is a stable configuration which is confirmed by the calculated phonon band structure. Then we focus on the effect on electronic properties due to the various hydrogen terminations and the related fundamental mechanism. We find that the electronic structures of GNM are very sensitive to edge hydrogen terminations. The unpassivated GNM is a magnetic halfsemimetal, the dangling bonds of hole edge could introduce the magnetic moment which causes the spin degeneracy to be eliminated. By a proper 21-H termination, the GNM is possible to be turned into a Bipolar Magnetic Semiconductor (BMS), which may be of great use in the next generation of information storage, the corresponding energy bands are also split due to the sublattice imbalance. For the other terminations such as mono- and di-hydrogenation, the GNM is non magnetic due to no dangling bonds and the balance of sublattice. We further find that the necessary condition of opening a sizable band gap is structure parameter $M=3 p$ no matter whether the mono- or di-hydrogenation, and di-hydrogenation could brings a larger gap than mono-hydrogenation owing to the stronger on-site potential between holes induced by di-hydrogenation. Furthermore, the gap width could be tuned between semimetallic and semiconducting states by varying the neck width. In short, our present work has solved an outstanding problem on how to properly pattern graphene to induce band gap, our findings could benefit further theoretical and experimental studies on the graphene-based nanostructures.

\section{Acknowledgements}

This work was supported by the National Natural Science Foundation of China (Grant No. 61371065 and 11674039), the Hunan Provincial Natural Science Foundation of China (Grant No. 2015JJ2009, 2015JJ2013, and 2015JJ3002), the Scientific Research Fund of Hunan Provincial Education Department (Grant No. 14A013 and 15A004), the Construct Program of the Key Discipline in Hunan Province, and the Aid Program for Science and Technology Innovative Research Team in Higher Educational Institutions of Hunan Province.

\section{References}

1 A. H. Castro Neto, F. Guinea, N. M. R. Peres, K. S. Novoselov and A. K. Geim, Rev. Mod. Phys., 2009, 81, 109-154.

2 W. Han and R. K. Kawakami, Phys. Rev. Lett., 2011, 107, 047207.

3 T. Nikolaos, J. Csaba, P. Mihaita, T. J. Harry and B. J. V. Wees, Nature, 2007, 448, 571-574.

4 A. K. Geim, Science, 2009, 324, 1530-1534.

5 K. S. Kim, Y. Zhao, H. Jang, S. Y. Lee, J. M. Kim, K. S. Kim, et al., Nature, 2009, 457, 706-710.

6 O. V. Yazyev and S. G. Louie, Nat. Mater., 2010, 9, 806-809.

7 Z. Zheng, C. Zhao, S. Lu, Y. Chen, Y. Li, H. Zhang and S. Wen, Opt. Express, 2012, 20, 23201-23214.

8 H. Zhang, Q. Bao, D. Tang, L. Zhao and K. Loh, Appl. Phys. Lett., 2009, 95, 141103.

9 B. Richard, J. Bjarke, N. Louis, A. Mie, R. Emile, B. Marco, et al., Nat. Mater., 2010, 9, 315-319.

10 L. Ci, Z. Xu, L. Wang, W. Gao, F. Ding, K. F. Kelly, et al., Nano Res., 2008, 1, 116-122.

11 P. Marco, R. Stefano, M. S. Polina, V. Sergio, E. Markus, P. Daniela, et al., ACS Nano, 2012, 6, 199-204.

12 M. Weinberg, C. Staarmann, C. Ölschläger, J. Simone and K. Sengstock, 2D Mater., 2016, 3, 024005.

13 T. G. Pedersen, C. Flindt, J. Pedersen, N. A. Mortensen, A. P. Jauho and K. Pedersen, Phys. Rev. Lett., 2008, 100, 136804.

14 J. Bai, X. Zhong, S. Jiang, Y. Huang and X. Duane, Nat. Nanotechnol., 2010, 5, 190-194.

15 W. Liu, Z. F. Wang, Q. W. Shi, J. Yang and F. Liu, Phys. Rev. B: Condens. Matter Mater. Phys., 2009, 80, 233405.

16 X. Ji, J. Zhang, Y. Wang, H. Qian and Z. Yu, Nanoscale, 2013, 5, 2527-2531.

17 R. Petersen, T. G. Pedersen and A. P. Jauho, ACS Nano, 2011, 5, 523-529.

18 M. D. Fischbein and M. Drndic, Appl. Phys. Lett., 2008, 93, 113107.

19 L. Z. Liu, S. B. Tian, Y. Z. Long, W. X. Li, H. F. Yang, J. J. Li, et al., Vacuum, 2014, 105, 21-25.

20 Y. Zhang, Y. Gao, L. Q. Liu, N. Xi, Y. C. Wang, L. P. Ma, et al., Appl. Phys. Lett., 2012, 101, 213101.

21 J. Li, R. J. Zhang, H. Q. Jiang and G. J. Cheng, Nanotechnology, 2011, 22, 475303-475309.

22 S. Rasappa, J. M. Caridad, L. Schulte, A. Caglianl, D. Borah, M. A. Momis, et al., RSC Adv., 2015, 5, 66711-66717. 
23 X. Liang, Y. S. Jung, S. Wu, A. Ismach, D. L. Olynick, S. Cabrini, et al., Nano Lett., 2010, 10, 2454-2460.

24 L. Liu, Y. L. Zhang, W. L. Wang, C. Z. Gu, X. D. Bai and E. G. Wang, Adv. Mater., 2011, 23, 1246-1251.

25 F. Ouyang, S. Peng, Z. Yang, Y. Chen, H. Zou and X. Xiong, Phys. Chem. Chem. Phys., 2014, 16, 20524-20531.

26 H. Şahin and S. Ciraci, Phys. Rev. B: Condens. Matter Mater. Phys., 2011, 84, 035452.

27 S. K. Singh, M. N. Amal and F. M. Peeters, J. Chem. Phys., 2014, 140, 074304.

28 D. M. Chen, P. M. Shenai and Y. Zhao, Phys. Chem. Chem. Phys., 2011, 13, 1515-1520.

29 M. Dvorak, W. Oswald and Z. Wu, Sci. Rep., 2013, 3, 2289.

30 R. Petersen and T. G. Pedersen, J. Phys.: Condens. Matter, 2015, 27, 225502.

31 W. Oswald and Z. Wu, Phys. Rev. B: Condens. Matter Mater. Phys., 2012, 85, 115431.
32 G. P. Tang, Z. H. Zhang, X. Q. Deng, Z. Q. Fan and H. L. Zhu, Phys. Chem. Chem. Phys., 2015, 17, 638-643.

33 J. Lee, A. K. Roy, J. L. Wohlwend, V. Varshney, J. B. Ferguson, W. C. Mitchel, et al., Appl. Phys. Lett., 2013, 102, 203107.

34 G. P. Tang, Z. H. Zhang, X. Q. Deng, Z. Q. Fan, Y. C. Zeng and J. C. Zhou, Carbon, 2014, 76, 348-356.

35 K. He, G. D. Lee, A. W. Robertson, E. Yoon and J. H. Warner, Nat. Commun., 2014, 5, 3040.

36 T. Wassmann, A. P. Seitsonen, A. M. Saitta, M. Lazzeri and F. Mauri, Phys. Rev. Lett., 2008, 101, 096402.

37 P. Wagner, V. V. Ivanovskaya, M. Melle-Franco, B. Humbert, J.-J. Adjizian, P. R. Briddon, et al., Phys. Rev. B: Condens. Matter Mater. Phys., 2013, 88, 094106.

38 O. V. Yazyev, Acc. Chem. Res., 2013, 46, 2319-2328.

39 E. H. Lieb, Phys. Rev. Lett., 1989, 62, 1201. 